\title{
The Deca-parsec Scale Radio Structures of BL Lac Objects
}

\author{
Redmond Hallahan* \\ University College Cork \\ E-mail: rhallahan@mars.ucc.ie

\section{Denise Gabuzda} \\ University College Cork \\ E-mail: gabuzda@physics.ucc.ie
}

\begin{abstract}
We present some results from our study of the 34 BL Lac objects in the 1-Jy sample defined by Kühr \& Schmidt [4]. All of the sources were observed using the VLBA at 4 frequencies near $1.5 \mathrm{GHz}$ in January 2004 and 4 were also observed in December 1999 at 1.6 and $2.3 \mathrm{GHz}$. The morphology and polarization structure on these scales can give us a more complete picture of how the structure of these objects extends from the parsec to kiloparsec scales. Our multi-epoch, multi-frequency polarization observations allow us to investigate the Faraday rotation present in each source and to comment on its stability on these scales over time. In the majority of sources, the jet polarization angles are aligned with the jet direction, right to the edge of the polarized flux. The spine-sheath polarization structures observed in the VLBI jets of a number of these sources are consistent with the presence of helical magnetic (B) fields[5]. Some of our rotation measure (RM) maps display transverse gradients in the inner jet, interpreted as further evidence for the presence of helical $\mathbf{B}$ fields in these jets[2]. The degree of polarization tends to increase away from the core and towards the jet edges, again consistent with helical jet $\mathbf{B}$ fields[5].
\end{abstract}

The 9th European VLBI Network Symposium on The role of VLBI in the Golden Age for Radio Astronomy and EVN Users Meeting

September 23-26, 2008

Bologna, Italy

\footnotetext{
*Speaker.
} 


\section{Introduction}

The first BL Lac object was discovered in 1929 and like many Active Galactic Nuclei (AGN), was originally classified as a faint variable star. They are a subclass of blazars, together with optically violent variable quasars, and are believed to have FRI host galaxies. They are distinguished by an absence of strong optical emission lines and often strong variability on parsec scales. In the AGN unification paradigm, BL Lac objects (and other compact radio loud AGN) eject bipolar jets with relativistic velocities from a central engine, thought to consist of a black hole and accretion disk. Blazars emit over many orders of magnitude of the EM spectrum, with the bulk of the emission attributed to synchrotron radiation emitted by electrons accelerated to relativistic speeds. Due to the high velocity of the emitting region, radiation emitted isotropically in the rest frame of the electrons appears to be confined to a cone of angle $\frac{1}{\Gamma}$ in the observer frame, where $\Gamma$ is the bulk Lorentz factor. Therefore one jet is 'boosted' towards the observer, while the other appears diminished or is undetected. In BL Lacs the jets are believed to be aligned close to our line of sight, leading to strong beaming and high Doppler factors. On larger scales the jets are slowed due to interaction with the external medium and appear less variable than on parsec scales, which probe closer to the central engine. Synchrotron radiation is linearly polarized and by measuring the degree of polarization, constraints can be placed on the degree of ordering of the magnetic field in the emitting region. The jets of BL Lac objects display a number of properties that are consistent with their jets having helical B fields, including gradients of the Faraday rotation measure (RM) across the jet, interpreted as being due to the systematic change in the line of sight component of the helical field across the jet (e.g. [2, 3, 6] and Mahmud \& Gabuzda, these proceedings).

Relatively few VLBI polarization observations of AGN have been carried out at $1.6 \mathrm{GHz}$. Such observations provide information about intermediate scales between the more common VLA (typically $\sim$ kiloparsec) and shorter wavelength VLBA $(\sim$ parsec) scales. Indeed, our observations sometimes display surprisingly different structure at these low frequencies when compared to higher frequency VLBI or VLA images.

\section{Observations and Analysis}

The images considered here were made from VLBA data obtained on December 13, 1999 and January 16-17, 2004. The 2004 observations were at 1.35, 1.43, 1.48 and $1.67 \mathrm{GHz}$ (all 34 BL Lacs in the sample of Kühr \& Schmidt [4]), while the 1999 observations were at 1.67 and $2.27 \mathrm{GHz}$ (the 5 sources $0735+178,0954+658,1732+389,1803+784$ and BL Lac). All observations involved all ten antennas, with the VLBA operating in snapshot mode. The data were calibrated and imaged in the NRAO AIPS package using standard techniques, with Los Alamos as the reference antenna.

\subsection{Polarization calibration}

The D-term calibration for the 2004 data was carried out using the compact sources $0745+241$ for 16 January and $0851+202$ for January 17 , solving simultaneously for the source and antenna polarization. The D-term calibration for the 1999 data was performed using the radio galaxy 3C84, which is unpolarized at these low frequencies. The electric vector position angle (EVPA) calibration for the 2004 data was carried out using VLA integrated polarization measurements for 
0851+202 (OJ287) made within 1 month of the VLBA observations. Since simultaneous VLA observations were not available for the 1999 data, we initially applied the $20041.67 \mathrm{GHz}$ EVPA calibration to the $19991.67 \mathrm{GHz}$ data. Note that EVPA calibration for a given reference antenna is typically stable over intervals as long as several years (see the MOJAVE website[9]).The polarization images for the 5 sources were very similar to the 2004 images, but showed a clear systematic offset of $15^{\circ}$ in the EVPAs of optically thin jet regions, consistent for all 5 sources. The $1.67 \mathrm{GHz}$ EVPA calibration for the 1999 data was corrected in accordance with this $15^{\circ}$ offset. The EVPA calibration for the $19992.27 \mathrm{GHz}$ data was determined analogously, by comparing the 2.27 and $1.67 \mathrm{GHz}$ images in regions known to have negligible Faraday rotation.

\subsection{Faraday Rotation}

When analysing low frequency data, it is especially important to take into account Faraday rotation of the plane of linear polarization, which occurs when electromagnetic radiation passes through a magnetised medium. It is characterised by a linear relationship between the EVPA and the square of the observing wavelength, with the coefficient of proportionality called the Rotation Measure (RM): $\Delta \chi \propto \lambda^{2} \int N_{e} B_{\|} d l$, i.e., the integral of the line of sight magnetic field, $B_{\|}$, times the electron density, $N_{e}$, along the line of sight. There are two sources of this rotation that must be considered: Faraday rotation occurring within or near the AGN and Faraday rotation occurring somewhere along our line of sight, the latter usually dominated by the ISM of our own Galaxy. The EVPAs for all sources were corrected for this Galactic Faraday rotation using the integrated RM values of Pushkarev [8] when the values given were greater than two sigma. Any remaining Faraday rotation is assumed to be intrinsic to the source.

\section{Results and Discussions}

\subsection{Morphology, polarization structure \& variability}

Our sample of 34 BL Lacs contains both compact and highly extended sources with corresponding polarization structure. We are primarily interested in sources showing extended polarization structure, though core polarization and RM values are important when comparing with higher frequency VLBI maps. Figure 1 shows three extended sources: 1538+149, 1803+784 and BL Lac. The jet polarization of all three sources shows signs of a spine-sheath structure, with a central region of transverse B field surrounded by regions of longitudinal B field, consistent with the jets having helical fields [1,5]. Recall that the observed EVPA is orthogonal to $\mathbf{B}$ in optically thin regions such as the VLBI jet and parallel to $\mathbf{B}$ in optically thick regions.

The jet of $1538+149$ stretches to $4.2 \mathrm{kpc}$ from the core, with signs of instability in the primarily eastwards jet direction. It shows strong core polarization with a complicated jet polarization structure centred around a knot approximately 50 mas from the core.

$1803+784$ has a rich polarization structure, observed at both epochs at $1.67 \mathrm{GHz}$ and also at 2.27 GHz in 1999. The morphology and jet EVPA showed no variation between epochs, while the core showed evidence of an additional polarized component in the later observations. This source illustrates both a distinctive spine of polarization longitudinal to the jet and a transverse sheath towards the edge. 


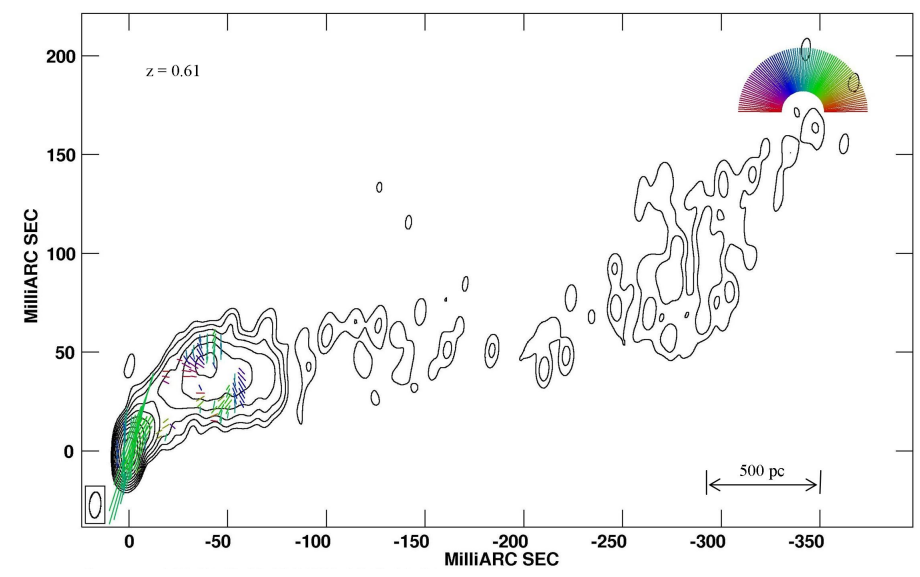

(a)

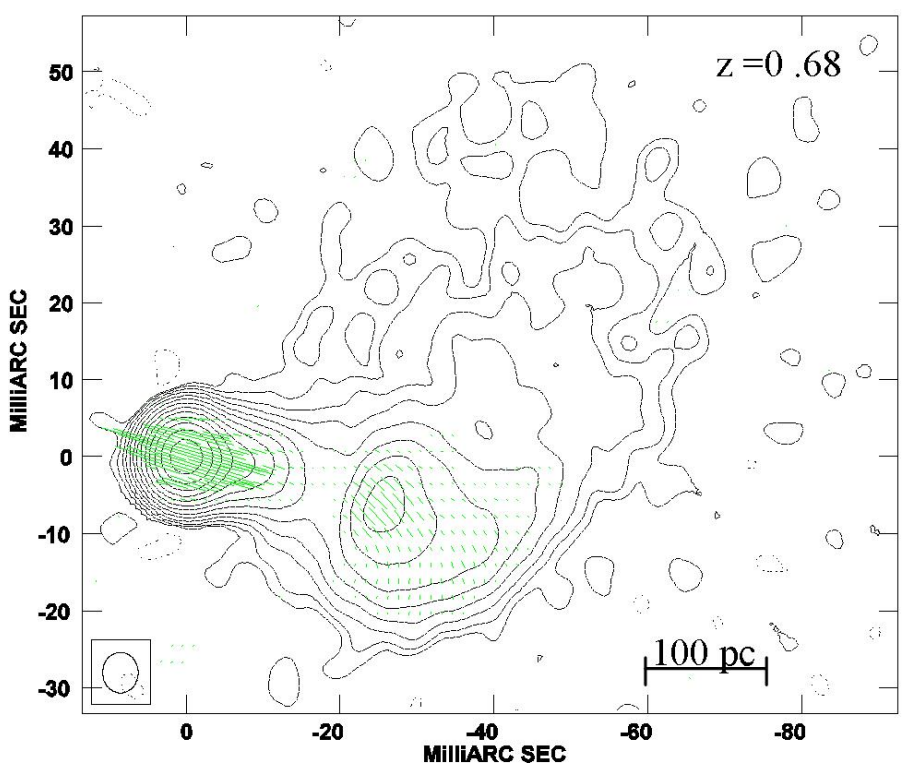

(b)

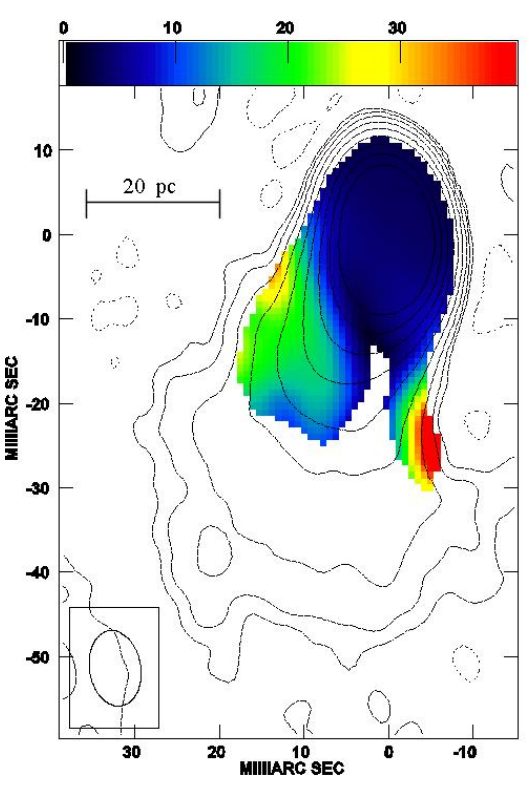

(c)

Figure 1: $1.67 \mathrm{GHz}$ total intensity maps with EVPA vectors for (a) $\mathbf{1 5 3 8 + 1 4 9}$ and (b) $\mathbf{1 8 0 3 + 7 8 4}$ (January 2004), and with fractional polarization for (c) BL Lac (December 1999).

Figure 1c shows how the fractional polarization in the jet of BL Lac increases both with distance from the core and towards the jet edges. This feature is also observed for several other sources in the sample.

Figure 2 shows three images of $0735+178$. This was the only one of the 5 sources observed at both epochs to show appreciable changes in jet polarization. The region concerned displays a complex RM structure of moderate strength in a source with almost zero integrated RM. The observed change in polarization is consistent with a decrease in the strength of a longitudinal $\mathbf{B}$ field component (or conversely, an increase in the strength of a transverse component) roughly 10 mas from the core. The degree of linear polarization increases towards the northern side of the jet. 


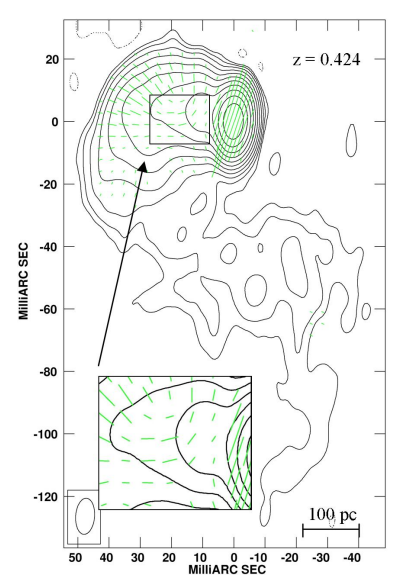

(a)

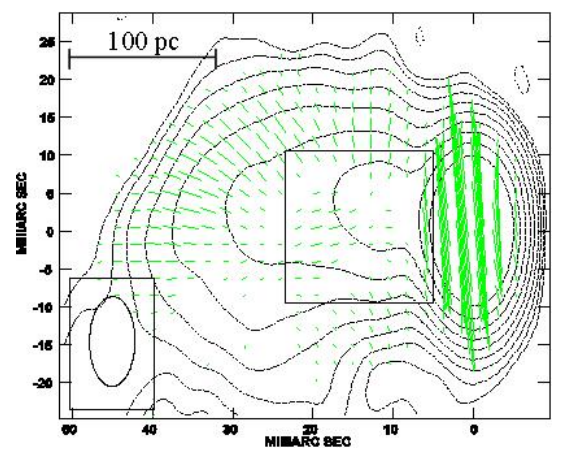

(b)

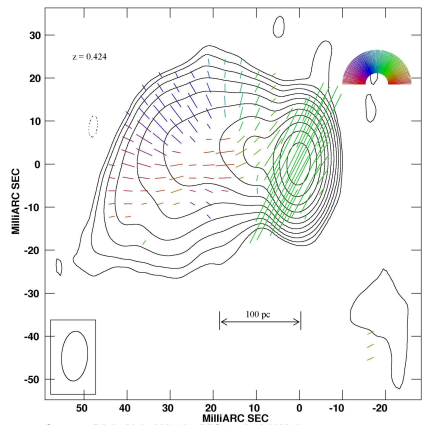

(c)

Figure 2: Total intensity images of $\mathbf{0 7 3 5 + 1 7 8}$ with EVPA vectors at $1.67 \mathrm{GHz}$ in (a) 2004 and (b) 1999 and (c) at $2.27 \mathrm{GHz}$ in 1999. (Corrected for integrated RM but not source RM so that epochs may be compared.)

\subsection{Rotation measure maps and gradients}

The RM distributions for some of the more extended sources can be complex. More compact objects often display transverse gradients across their cores. Figure 3 shows three such sources, $0716+714,0954+568$ and $1749+701$. The values for the RM are usually some tens of $\mathrm{rad} / \mathrm{m}^{2}$.

The gradients in all of these sources have also been observed at higher frequencies using the VLBA and the direction of the RM gradients are the same in all cases([7] and Mahmud \& Gabuzda, these proceedings). When two opposite gradients were observed at higher frequencies, it is the gradient further from the core that we observe at $1.6 \mathrm{GHz}$; in the case of $1749+701$, we detect the core-region gradient reported by Mahmud \& Gabuzda (these proceedings) as well as an oppositely directed gradient further from the core (see their discussion of this phenomenon); note that the parsec-scale jet extends to the Northwest. We have ensured that these gradients are not dependent upon a particular beam size, nor are they sensitive to the core shift, which must be taken into account before making spectral index maps.

\section{Conclusion}

The rich intensity and polarization structures in the images presented here are fairly typical for the BL Lacs in the Kühr \& Schmidt 1-Jy sample. We find that the decaparsec-scale jets of BL Lac objects are highly stable structures, displaying very little variation outside of the $1.6 \mathrm{GHz}$ VLBI core over the course of $\sim 4.5$ years. Changes in the core EVPAs of all objects were observed, as is to be expected for this partially optically thick and unresolved region, where the effect of emerging components may alter both the observed EVPA and polarized intensity. Indeed, on these scales, changes in the core polarization and RM structure offer our best method of comparison with higher frequency observations. The observed polarization structures are consistent with helical jet 


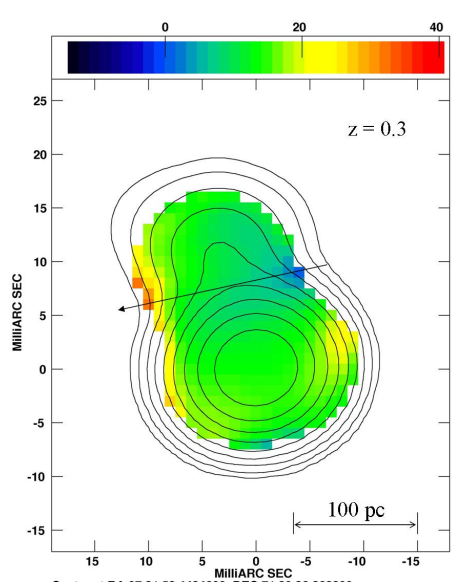

(a)

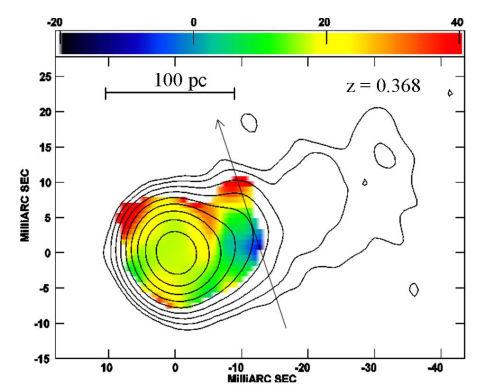

(b)

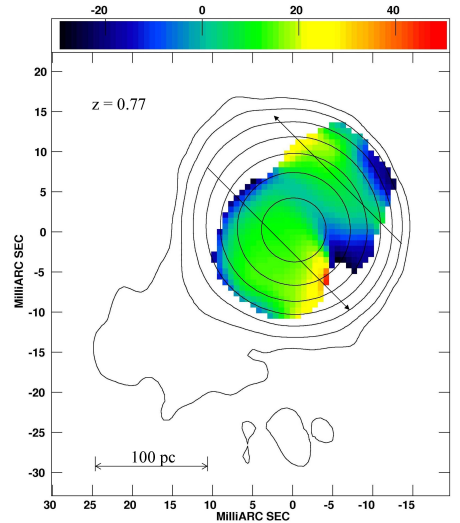

(c)

Figure 3: 2004 RM maps for (a) 0716+714, (b) 0954+568, and (c) 1749+701.

B fields, in particular spine-sheath structures, with orthogonal $\mathbf{B}$ field near the jet ridge line and longitudinal $\mathbf{B}$ field near the jet edges are observed in many sources.

Both the fractional polarization distributions and transverse RM gradients observed in some sources represent tell-tale signs of a helical jet $\mathbf{B}$ fields: the gradient in the Faraday rotation is due to the systematic change in the line of sight $\mathbf{B}$ field across the jet. Such transverse RM gradients have also been observed for a number of BL Lac objects between 5 and $43 \mathrm{GHz}$ [3]. The gradients we have observed are in the same direction as those found in the literature, demonstrating that these gradients are not artefacts and are common features in the jets of BL Lacs.

This publication has emanated from research supported by the Research Frontiers Programme of Science Foundation Ireland. We thank P. Veres for his work on the initial calibration of the 2004 data. The National Radio Astronomy Observatory is operated by Associated Universities Inc.

\section{References}

[1] Gabuzda, D. C., 2006, Proc. $8^{\text {th }}$ EVN Symposium, Ed. A. Marecki, http://pos.sissa.it//archive/conferences/036/011/8thEVN_011.pdf

[2] Gabuzda, D. C., Murray, E. \& Cronin, P. 2004, MNRAS, 351, L89

[3] Gabuzda, D. C., Vitrishchak, V. M., Mahmud, M. \& O'Sullivan, S. P. 2008, MNRAS, 384, 1003

[4] Kühr, H. \& Schmidt, G. D. 1990, AJ, 99,1

[5] Lyutikov, M.; Pariev, V. I. \& Gabuzda, D. C. 2005, MNRAS, 360, 869

[6] Mahmud, M. \& Gabuzda, D. C. 2008, in "Extragalactic Jets: Theory \& Observation from Radio to Gamma Rays", Ed. Travis A. Rector \& David S. De Young, ASP Conf. Series, 386, p494

[7] O'Sullivan, S. P. \& Gabuzda, D. C. 2009, MNRAS, in press

[8] Pushkarev, A. 2001, Astron. Rep., 45, 667

[9] M.O.J.A.V.E Program website, http://www.physics.purdue.edu/MOJAVE/ 